\title{
Therapeutic potentials of naringin on polymethylmethacrylate induced osteoclastogenesis and osteolysis, in vitro and in vivo assessments
}

\author{
This article was published in the following Dove Press journal: \\ Drug Design, Development and Therapy \\ 9 December 2013 \\ Number of times this article has been viewed
}

\author{
Nianhu $\mathrm{Li}^{1,2, *}$ \\ Zhanwang $\mathrm{Xu}^{2, *}$ \\ Paul H Wooley ${ }^{1,3}$ \\ Jianxin Zhang ${ }^{2}$ \\ Shang-You Yang 1,3 \\ 'Department of Surgery, \\ Orthopedics, University of Kansas \\ School of Medicine, Wichita, KS, \\ USA; ${ }^{2}$ Department of Orthopedics, \\ Affiliated Hospital to Shandong \\ University of Traditional Chinese \\ Medicine, Jinan, People's Republic \\ of China; ${ }^{3}$ Orthopaedic Research \\ Institute, Via Christi Wichita Hospitals, \\ Wichita, KS, USA
}

*The first two authors contributed equally to this work

\begin{abstract}
Wear debris associated periprosthetic osteolysis represents a major pathological process associated with the aseptic loosening of joint prostheses. Naringin is a major flavonoid identified in grapefruit. Studies have shown that naringin possesses many pharmacological properties including effects on bone metabolism. The current study evaluated the influence of naringin on wear debris induced osteoclastic bone resorption both in vitro and in vivo. The osteoclast precursor cell line RAW 264.7 was cultured and stimulated with polymethylmethacrylate (PMMA) particles followed by treatment with naringin at several doses. Tartrate resistant acid phosphatase (TRAP), calcium release, and gene expression profiles of TRAP, cathepsin $\mathrm{K}$, and receptor activator of nuclear factor-kappa $\mathrm{B}$ were sequentially evaluated. PMMA challenged murine air pouch and the load bearing tibia titanium pin-implantation mouse models were used to evaluate the effects of naringin in controlling PMMA induced bone resorption. Histological analyses and biomechanical pullout tests were performed following the animal experimentation. The in vitro data clearly demonstrated the inhibitory effects of naringin in PMMA induced osteoclastogenesis. The naringin dose of $10 \mu \mathrm{g} / \mathrm{mL}$ exhibited the most significant influence on the suppression of TRAP activities. Naringin treatment also markedly decreased calcium release in the stimulated cell culture medium. The short-term air pouch mouse study revealed that local injection of naringin ameliorated the PMMA induced inflammatory tissue response and subsequent bone resorption. The long-term tibia pin-implantation mouse model study suggested that daily oral gavage of naringin at $300 \mathrm{mg} / \mathrm{kg}$ dosage for 30 days significantly alleviated the periprosthetic bone resorption. A significant increase of periprosthetic bone volume and regaining of the pin stability were found in naringin treated mice. Overall, this study suggests that naringin may serve as a potential therapeutic agent to treat wear debris associated osteolysis.
\end{abstract}

Keywords: naringin, osteoclastogenesis, aseptic loosening, periprosthetic osteolysis

\section{Introduction}

Osteolysis represents a major pathological process that occurs during aseptic prosthetic loosening and osteoporosis, and is characterized by regional bone loss, inflammation, and bone demineralization. Total joint replacement is a successful surgical procedure to restore joint function and to repair joints damaged by traumatic injury or arthritis. Although failure of total joint replacement due to infection and surgical error has been greatly reduced in recent years, aseptic prosthetic loosening remains the most common complication and represents a major problem for the long-term success and survival of prostheses. ${ }^{1}$ It appears that wear debris generated at the bone-implant
Correspondence: Shang-You Yang Orthopaedic Research Institute,Via Christi Wichita Hospitals, 929 N St. Francis Street, Wichita, KS, USA 67260

Tel + I 3162685455

Fax + I 316 29| 4998

Email shang-you.yang@wichita.edu
Drug Design, Development and Therapy 2014:8 I-II

Dovepress

http://dx.doi.org// 0.2147/DDDT.S52714 (c) (i) (5) 2014 Li et al. This work is published by Dove Medical Press Limited, and licensed under Creative Commons Attribution - Non Commercial (unported, v3.0) License. The full terms of the license are available at http://creativecommons.org/licenses/by-nc/3.0/. Non-commercial uses of the work are permitted without any further permission for the License are administered by Dove Medical Press Limited. Information on how to request permission may be found at: http://www.dovepress.com/permissions.php 
interface plays a critical role in initiating local inflammation and periprosthetic bone loss, leading to the ultimate failure of the prostheses. ${ }^{2}$

Studies have suggested that the receptor activator of nuclear factor-kappa B ligand (RANKL)/osteoprotegerin pathway plays a key role in bone metabolism and osteolysis. ${ }^{3}$ RANKL, expressed by macrophages, osteoblasts, marrow stromal cells, and lymphocytes, binds to its physiological receptor, receptor activator of nuclear factor-kappa B (RANK), on the cell surface of osteoclasts and osteoclast precursors, resulting in proliferation, differentiation, and maturation of osteoclasts and subsequent local osteolysis. ${ }^{4}$ Mice genetically deficient for RANKL or RANK often exhibit severe osteopetrosis, ${ }^{5}$ indicating that RANKL and RANK are essential for osteoclast development. Osteoclasts are characterized by high expression of tartrate resistant acid phosphatase (TRAP) and cathepsin K (CPK), gene products that contribute to the mineral dissolution and resorption of the organic matrix of bone. ${ }^{6,7}$

Naringin is a compound extracted from citrus fruits such as grapefruit and is reported to be beneficial in the treatment and prevention of osteoporosis. ${ }^{8}$ In vitro studies have revealed that naringin actively promoted the differentiation of mesenchymal stem cells to osteogenic cells. ${ }^{9}$ An in vivo study ${ }^{10}$ reported that naringin suppressed osteoclast formation, while another study using osteoclast precursor cells showed that naringin abrogated osteoclastogenesis and bone resorption via inhibition of RANK induced nuclear factorkappa B (NF- $\mathrm{KB})$ and extracellular signal-regulated kinase (ERK) activation. ${ }^{11}$ However, there is lack of knowledge on naringin's therapeutic influence on particulate wear debris associated osteolysis.

We hypothesized that naringin may inhibit osteoclastogenesis and exert therapeutic effects on wear debris associated osteolysis. Using both in vitro and in vivo experimental approaches, this study has tested naringin's possible therapeutic effects and its efficacy in polymethylmethacrylate (PMMA) induced inflammation, osteoclastogenesis, and osteolysis.

\section{Materials and methods}

\section{Particle preparation}

PMMA particles with a mean diameter of $0.33 \mu \mathrm{m}$ $(0.33 \pm 0.019 \mu \mathrm{m})$ were obtained from a commercial source (Polysciences, Warrington, PA, USA). The size and distribution of the particles were evaluated with a Coulter particle counter (Beckman Coulter, Inc., Indianapolis, IN, USA) and by scanning electron microscopy (SEM; Hitachi S-2400, Japan). ${ }^{12}$ The particles were washed in $70 \%$ ethanol solution and heat

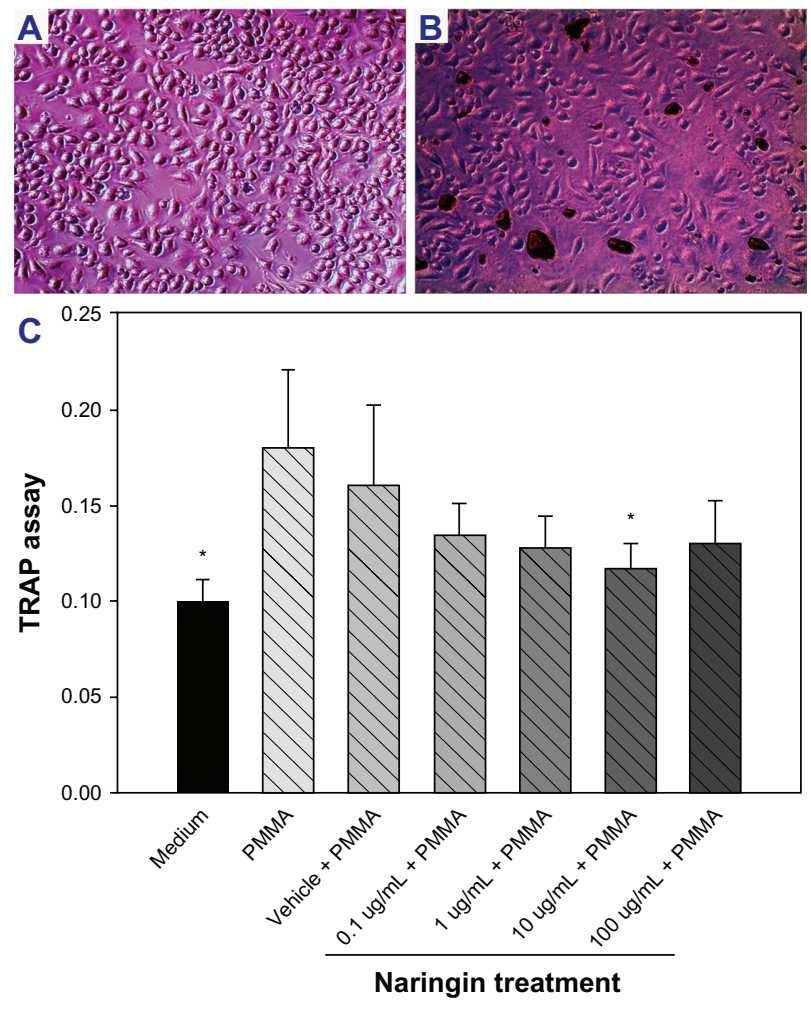

Figure I RAW 264.7 cell culture stimulation and trap assay.

Notes: (A) RAW 264.7 cells cultured (200×) and (B) RAW 264.7 cells stimulated with polymethylmethacrylate (PMMA) particles (200x). (C) PMMA particle stimulation significantly increases RAW 264.7 cell TRAP function while naringin inhibited it in a concentration dependent manner. A concentration of $10 \mu \mathrm{g} / \mathrm{mL}$ worked best for the inhibition. *Denotes $P<0.05$ when compared to PMMA.

Abbreviations: PMMA, polymethylmethacrylate; TRAP, tartrate resistant acid phosphatase.

sterilized. A limulus assay (Endosafe; Charles Rivers Laboratories, Charlestown, SC, USA) was then performed on particles to ensure they were free of endotoxin. Particles were suspended in sterile phosphate-buffered saline (PBS) and stored at $4^{\circ} \mathrm{C}$.

\section{RAW 264.7 cell cultures stimulation and TRAP assay}

The mouse RAW 264.7 macrophage cell line (American Type Culture Collection, Manassas, VA, USA) was used for in vitro assessment of the naringin effects. RAW 264.7 cells were seeded on 8-chamber slides in Dulbecco's Modified Eagle's Medium (DMEM) containing 10\% fetal bovine serum and antibiotics (100 units $/ \mathrm{mL}$ penicillin $+100 \mu \mathrm{g} /$ $\mathrm{mL}$ streptomycin) at $37^{\circ} \mathrm{C}$ in a humidified incubator with $5 \%$ $\mathrm{CO}_{2}$. PMMA particles in $5 \mathrm{mg} / \mathrm{mL}$ were added to a 96-well plate with $1 \times 10^{5}$ RAW 264.7 cells/well. For treatment groups, naringin was added along with PMMA suspension concentrations ranging from $0.1 \mu \mathrm{g} / \mathrm{mL}$ to $100 \mu \mathrm{g} / \mathrm{mL}$. RANKL $(10 \mathrm{ng} / \mathrm{mL})$ was added to wells of the positive control group. Cell culture media were collected after 3 days culture for 
TRAP activity determination as described elsewhere. ${ }^{13}$ In brief, $20 \mu \mathrm{L}$ of supernatant from RAW 264.7 cell culture medium was added to a 96 -well plate and $80 \mu \mathrm{L}$ of freshly prepared reaction buffer $(0.33 \mathrm{~mol} / \mathrm{L}$ acetic acid, $0.167 \%$ Triton X-100, $0.33 \mathrm{~mol} / \mathrm{L}$ sodium chloride, $3.33 \mathrm{~mol} / \mathrm{L}$ ethylenediaminetetraacetic acid at $\mathrm{pH} 5.5,1.5 \mathrm{mg} / \mathrm{mL}$ of ascorbic acid, $7.66 \mathrm{mg} / \mathrm{mL}$ of sodium tartrate, $3 \mathrm{mg} / \mathrm{mL}$ of 4-nitrophenylphosphate) was added. The reaction was left for 1 hour at $37^{\circ} \mathrm{C}$ in the dark, and then stopped with $100 \mu \mathrm{L}$ of $0.3 \mathrm{~mol} / \mathrm{L}$ sodium hydroxide. The colorimetric changes were measured at $405 \mathrm{~nm}$ with $650 \mathrm{~nm}$ as the reference using a microplate spectrophotometer (Molecular Devices LLC, Sunnyvale, CA, USA). Meanwhile, the cells were fixed in buffered acetone and processed for histochemical TRAP staining using a commercial kit (387A-1 KT; Sigma-Aldrich, St Louis, MO, USA) as detailed previously. ${ }^{14}$

\section{Real time quantitative polymerase chain reaction (PCR)}

RAW 264.7 cells were cultured in the presence of $1 \%$ PMMA particles for 6 hours before collection for RNA extraction. For naringin interference experiments (dimethyl sulfoxide [DMSO] was used as vehicle to dissolve the compound), RAW 264.7 cells were pretreated with naringin for 3 hours before addition of PMMA particles. Real time quantitative PCR was carried out according to the manufacturer's instructions (StepOnePlus Real-Time PCR System; Life Technologies, Carlsbad, CA, USA). Primers used in this study were as follows: TRAP (Cat\# QT00131012; QIAGEN Inc., Valencia, CA, USA); RANK, 5'-GGGTGGGGGGCAGACTTCAC-3' (forward), 5'-ATGCCAGCAGCCTGCACCAG-3' (reverse); CPK, 5'-CGTGCAGCAGAACGGAGGCA-3' (forward), 5'-TAGCTGCCTTTGCCGTGGCG-3' (reverse).

To standardize the target gene level with respect to variability in RNA and cDNA, the housekeeping gene $18 \mathrm{~S}$ was used as an internal control. Normalization and analysis of the reporter signals $(\Delta R n)$ at the threshold cycle were recorded using the machine firmware.

\section{Establishment of the bone resorption air pouch model and naringin intervention}

All experimental procedures and protocols were approved by our Institutional Animal Care and Use Committee (IACUC). The air pouch model of bone resorption was established in our laboratories as described previously. ${ }^{15}$ Briefly, air pouches were generated by injection of sterile air on the back of female BALB/c mice ( 8 to 10 weeks of age). At day 6 after air pouch formation, a proximal or distal section of the femur, or a section of calvarium from the congeneric littermate donors, was surgically implanted into the established air pouches. Mice were randomly divided into four groups (six mice each) the next day. Three experimental groups of mice were given PMMA particle $(0.3 \mathrm{~mL}$ of $10 \mathrm{mg} / \mathrm{mL}$ stock) injection into each pouch to provoke inflammatory responses, and respectively received intrapouch injection of 1) $150 \mathrm{mg} / \mathrm{kg}$ of naringin in $0.2 \mathrm{~mL} \mathrm{DMSO}$; 2) $30 \mathrm{mg} / \mathrm{kg}$ naringin in $0.2 \mathrm{~mL}$ DMSO; and 3) $0.2 \mathrm{~mL}$ DMSO vehicle. The mice in the control group without PMMA stimulation received $0.2 \mathrm{~mL}$ of PBS. Mice were kept for 7 more days before being sacrificed in a $\mathrm{CO}_{2}$ chamber, and the whole pouches were surgically harvested. Pouch fluid plus $0.2 \mathrm{~mL}$ of rinsing saline was collected as pouch lavage for free calcium determination. The remainder of the pouch tissue with the intact bone-implant was either snap frozen for frozen tissue sectioning (calvarium embedded pouches for TRAP stain), or fixed in 10\% buffered formalin (Thermo Fisher Scientific, Waltham, MA, USA) for paraffin embedding.

\section{Establishment of the titanium-pin (Ti-pin) model on bone resorption and naringin intervention}

To study oral administration of naringin and its long-term influence on particle-induced periprosthetic bone resorption, a newly characterized mouse proximal tibia Ti-pin failure model was adopted. ${ }^{16}$ Female BALB/c mice 10 to 12 weeks old were used in this experiment, and all procedures were approved by Wichita State University IACUC. A flat-headed Ti-pin ( $5 \mathrm{~mm}$ long $\times 0.8 \mathrm{~mm}$ diameter, $12 \mathrm{~mm}$ flat top; specially manufactured by Stryker Orthopaedics, Inc., Mahwah, NJ, USA) was surgically inserted into the proximal tibia to a depth where the head of the pin was flush with the knee joint surface. At 3 weeks after surgery (when the implanted pin gained osteointegration), the mice were divided into three groups (six mice per group): two groups received daily intragastric gavages with high dose $(1500 \mathrm{mg} /$ $\mathrm{kg}$ body weight) or low dose $(300 \mathrm{mg} / \mathrm{kg})$ of naringin, while the third group of mice received PBS gavages as controls. At 4 weeks postsurgery, all animals were given an intra-articular injection of PMMA particles to the implanted knee joint to mimic prosthetic wear. MicroCT (SCANCO viva 4.0; Scanco Medical, Basserdorf, Switzerland) scanning was performed immediately after surgery, and at 3 weeks and 7 weeks after the surgery to monitor the morphological bone changes. The Scanco MicroCT Evaluation Program V6.5-1 software was used for data collection. The mice were sacrificed 7 weeks after surgery (4 weeks after naringin intervention), and the 
implant bearing limb collected for implant stability examination (pullout test) and histological assessments (peri-implant soft tissue formation, pit bone erosions, and bone collagen degradation). MicroCT images of the mouse proximal tibia around the pin were reconstructed and processed using a threshold of $300-700 \mathrm{mg} \mathrm{HA} / \mathrm{ccm}^{17}$ to calculate the BV/TV (bone volume/total volume) values of each specimen.

\section{Calcium release assay on mouse calvaria cultures and air pouch bone resorption model}

Stimulated medium was prepared by incubation of RAW 264.7 cells in the serum-free DMEM medium containing PMMA $(0.2 \mathrm{mg} / \mathrm{mL})$ for 3 days. The media were then harvested and stored at $-80^{\circ} \mathrm{C}$ until use. Calvaria were collected from 12 week old C57BL/6 mice and dissected free of soft tissue. Stimulated medium from activated RAW 264.7 cells was diluted (1:1) in serum-free DMEM and incubated with a piece of calvarium for 5 days. To investigate whether naringin can inhibit stimulated medium-induced bone resorption, calvaria were cultured in the presence of naringin $(10 \mu \mathrm{g} / \mathrm{mL})$ for up to 5 days. Bone cultures without naringin treatment were included as controls. At the end of the experiments, the calcium released into the media was measured by the Calcium (Arsenazo) Reagent Set (Pointe Scientific, Inc., Canton, MI, USA). ${ }^{18}$ Briefly, $10 \mu \mathrm{L}$ of medium was added to $190 \mu \mathrm{L}$ of $0.11 \mathrm{mM}$ o-cresolphthalein complexone/17 mM 8-hydroxyquinoline/976 mM 2-amino-2-methyl-1-propanol/2 mM potassium cyanide solution. The intensity of the calcium-ocresolphthalein complexone complex was assayed at $570 \mathrm{~nm}$ on a microplate spectrophotometer (Molecular Devices). An aqueous calcium solution $(10 \mathrm{mg} / \mathrm{dL}=2.5 \mathrm{mM})$ was used as standard. The calcium concentration was calculated using the following formula:

$\underset{(\text { mmoles })}{\text { Calcium }}=\frac{\text { Absorbance of sample }}{\text { Absorbance of standard }} \times \begin{aligned} & \text { Concentration of } \\ & \text { standard }(2.5 \mathrm{mM}) .\end{aligned}$

Pouch lavage samples from the bone resorption air pouch model experiment were also examined for free calcium concentration using the same procedure.

\section{Histochemical staining and image analysis of bone air pouch tissue}

Formalin-fixed pouches with implanted bone were decalcified with formic acid/sodium citrate before being paraffin-embedded and mounted with a consistent orientation and cut to
$6 \mu \mathrm{m}$ sections. The sections were stained with hematoxylin and eosin to examine bone erosion and changes in inflammatory parameters in pouch membranes. Histochemical TRAP staining was performed on frozen sectioned calvarium air pouch tissues to localize the osteoclast-like cells. ${ }^{15,19}$ Briefly, cryosections at $8 \mu \mathrm{m}$ thickness were prepared and fixed in buffered acetone for 30 seconds. Sections were incubated at $37^{\circ} \mathrm{C}$ for 1 hour in $100 \mathrm{mM}$ acetate buffer (pH 5.2), containing $0.5 \mathrm{mM}$ of naphthol AS-BI phosphoric acid, $2.2 \mathrm{mM}$ of Fast Garnet GBC, and $8 \mathrm{mM}$ of sodium tartrate (Sigma-Aldrich). The dark purple stained cells along the bone surfaces were quantified as TRAP positive $\left(\mathrm{TRAP}^{+}\right)$cells. $\mathrm{TRAP}^{+}$multinucleated cells containing more than three nuclei were counted as osteoclasts, whereas TRAP ${ }^{+}$ mononucleated cells were counted as preosteoclasts. A computerized image analysis system with the software "Image-Pro Plus" (Media Cybernetics, Silver Spring, MD, USA) was used to analyze the local inflammation (pouch membrane thickness and inflammatory cell infiltration) and local osteoclastogenesis (TRAP $^{+}$cell counts) as described previously. ${ }^{14,15,19,20}$

\section{Pin pullout testing}

Immediately following sacrifice of the animals receiving proximal tibia Ti-pin implantation, the mouse limb bearing the titanium implant was removed by disarticulating the knee joint. All soft tissue around the prosthetic joint was carefully removed to expose the implanted pin head surface and proximal tibia. A custom aluminum fixture was designed to securely hold and align the pin for extraction. The test fixture utilized two razor blades which were tightened onto the head of the pin to securely grip the pin for removal. The pin and tibia were mounted to this fixture and the pin alignment was checked to ensure that its axis was parallel with the direction of extraction and orthogonal to the razor blade grips. With the tibia pin held by the fixture and the fixture mounted on the load frame, approximately $1 \mathrm{~cm}$ of the distal portion of the tibia was then cemented into a potting box using high strength dental cement. The cement was given at least 30 minutes of cure time before testing. A Bose 3200 load frame was used to run the pin extraction test. The test was run under displacement control at a rate of $1 \mathrm{~mm} / \mathrm{min}$ and the actuator position and applied load were recorded as a function of time using Bose WinTest ${ }^{\circledR}$ software.

\section{Statistical analysis}

Alldata are presented as the mean \pm standard deviation, and single factor analysis of variance with LSD post hoc multiple comparison (IBM Corporation, Armonk, NY, USA) was used to assess the statistical significance among groups. The student's $t$-test (IBM) was adopted for statistical comparisons 
before and after treatment. In all statistical evaluations, $P<0.05$ was considered statistically significant.

\section{Results}

\section{Naringin inhibited the TRAP function of particle induced RAW 264.7 cells}

PMMA particle stimulation significantly increased the function of RAW 264.7 cell trap function when compared to the medium group. Naringin decreased the TRAP function with a wide range of concentrations, and the concentration of $10 \mu \mathrm{g} / \mathrm{mL}$ exhibited the most significant inhibition among all the concentrations tested (Figure 1).

\section{Naringin inhibition of PMMA induced RAW 264.7 cell osteoclastogenesis}

Addition of RANKL dramatically induced osteoclastogenesis of the RAW 264.7 cells in culture by significantly elevating TRAP activity and $\mathrm{TRAP}^{+}$staining (Figure 2B). Challenge with PMMA particles also promoted the RAW 264.7 cells to become TRAP $^{+}$and merge to multinucleated cells (Figure 2C). PMMA induced osteoclastogenesis was significantly inhibited by naringin treatment $(10 \mu \mathrm{g} / \mathrm{mL}$ for 3 days), with less multinucleated cell formation and fewer $\mathrm{TRAP}^{+}$cells (Figure 2E). Real time PCR data suggested that PMMA particles markedly increased the gene expression of TRAP, CPK, and RANK ( 3 to 4 fold) as illustrated in Figure 3 , whereas naringin treatment $(10 \mu \mathrm{g} / \mathrm{mL})$ significantly down regulated the messenger (m)RNA expression of TRAP, CPK, and had a trend to downregulate the mRNA expression of RANK.

\section{Naringin diminished osteoclastic calcium release induced by stimulated medium from the PMMA activated RAW \\ 264.7 cells and in the air pouch model}

Naringin treatment inhibited calcium release from the calvaria bone in stimulated medium. Coculture of the mouse calvaria with stimulated medium from PMMA particle activated RAW 264.7 cells resulted in a markedly elevated calcium level in the medium after 5 days $(166.47 \pm 35.19 \mu \mathrm{M})$ when compared to the controls cultured in serum-free DMEM medium $(86.81 \pm 3.75 \mu \mathrm{M})$. In the presence of naringin $(10 \mu \mathrm{g} / \mathrm{mL})$, a significant decrease in calcium release into culture medium was observed $(93.43 \pm 2.59 \mu \mathrm{M}, P<0.05)$.

Naringin treatment also inhibited calcium release from the implanted bone air pouch model. The concentrations of calcium in the lavage fluid averaged $135.32 \pm 19.00 \mu \mathrm{M}$ in the PMMA + PBS group, 137.42 $\pm 24.20 \mu \mathrm{M}$ in
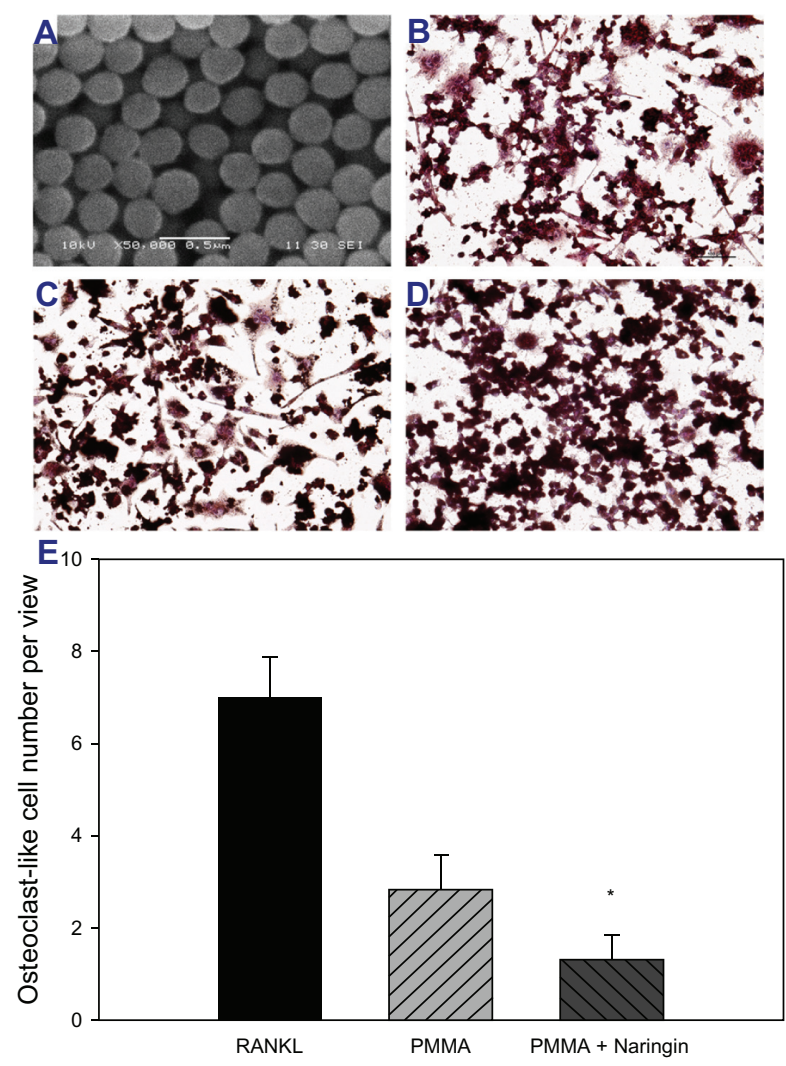

Figure 2 Naringin inhibited polymethylmethacrylate induced osteoclastogenesis of RAW 264.7 cells.

Notes: (A) Scanning electron micrograph image of polymethylmethacrylate (PMMA) particles used in the study $(50,000 \times)$. Tartrate resistant acid phosphatase (TRAP) stained RAW 264.7 cells (B) stimulated with receptor activator of nuclear factor-kappa B ligand; (C) stimulated with PMMA particles; and (D) stimulated with PMMA particles and treated with naringin ( $10 \mu \mathrm{g} / \mathrm{mL}$ for 3 days). (E) Quantification summary of the TRAP positive cells among the groups. *Denotes $P<0.05$ when compared with the PMMA group.

Abbreviations: PMMA, polymethylmethacrylate; RANKL, receptor activator of nuclear factor-kappa B ligand.

the PMMA + vehicle group, $95.61 \pm 18.26 \mu \mathrm{M}$ in the $\mathrm{PMMA}+30 \mathrm{mg} / \mathrm{kg}$ naringin group, and $50.12 \pm 15.94 \mu \mathrm{M}$ in the PMMA $+150 \mathrm{mg} / \mathrm{kg}$ naringin group, respectively. A dose of $150 \mathrm{mg} / \mathrm{kg}$ naringin significantly decreased calcium release when compared to the PMMA + vehicle group $(P<0.05)$.

\section{Naringin protected against bone resorption/degradation induced by PMMA particles in the air pouch model}

A macroscopic view of the air pouches at sacrifice illustrated that introduction of naringin dramatically ameliorated the inflammatory appearance (Figure 4A) when compared to the vehicle controls of the PMMA particle-challenged pouches which remained significantly inflammatory and erythematous (Figure 4B). Histological analysis of hematoxylin and eosin stained bone implanted pouch sections exhibited extensive inflammatory cellular infiltration and membrane hyperplasia in the PMMA group (Figure 4D). Naringin treatment sig- 


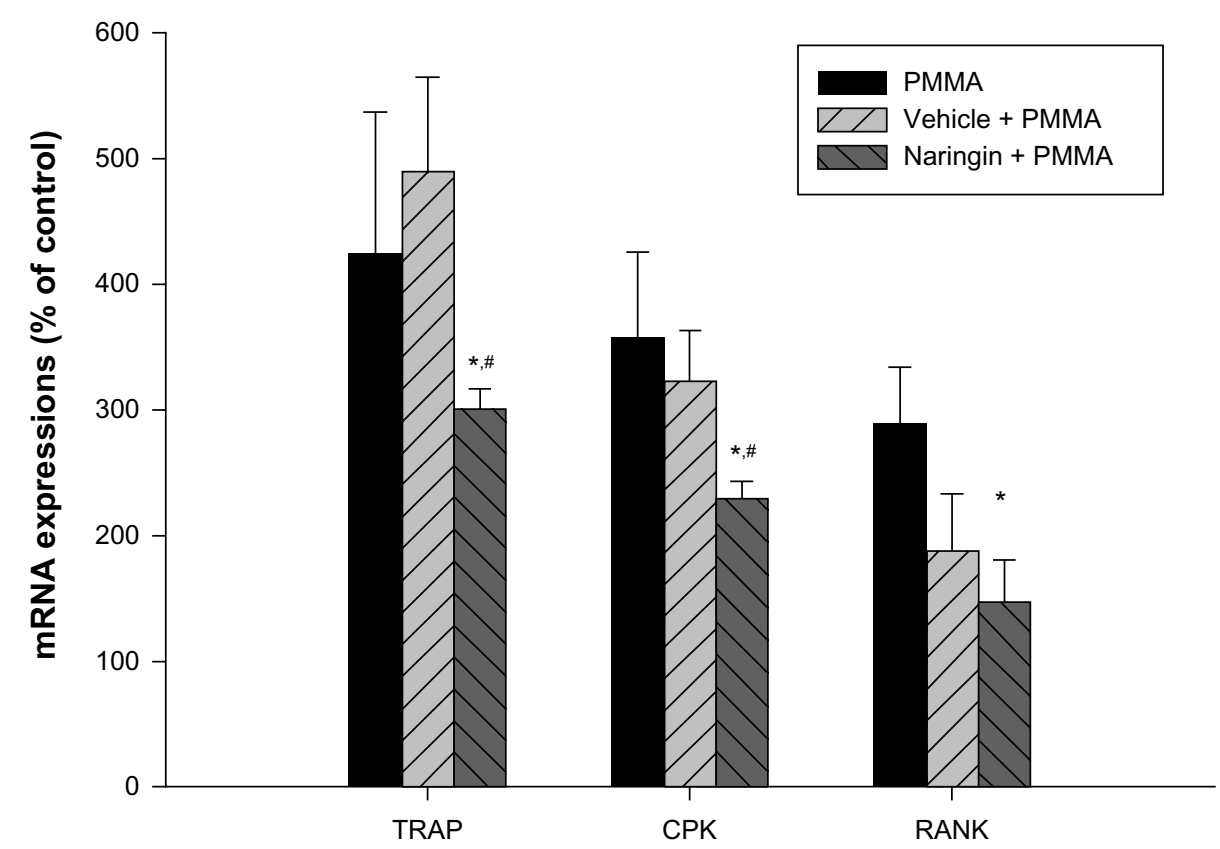

Figure 3 Gene expression profile of RAW 264.7 cells following polymethylmethacrylate stimulation and naringin treatment.

Notes: RAW 264.7 cells were preincubated with or without naringin $(10 \mu \mathrm{g} / \mathrm{mL})$ for 3 hours prior to the addition of I\% polymethylmethacrylate (PMMA) particles. After treatment for 6 hours, total RNA was isolated and gene expression was evaluated by real time polymerase chain reaction techniques. RAW 264.7 cells cultured in medium without naringin, vehicle, or particles were used as control. Data were collected in triplicate cultures of RAW 264.7 cells from three independent experiments. *Denotes $P<0.05$ when compared with the PMMA group; \#denotes $P<0.05$ when compared with the vehicle + PMMA group.

Abbreviations: CPK, cathepsin K; PMMA, polymethylmethacrylate; RANK, receptor activator of nuclear factor-kappa B; TRAP, tartrate resistant acid phosphatase; mRNA, messenger RNA.
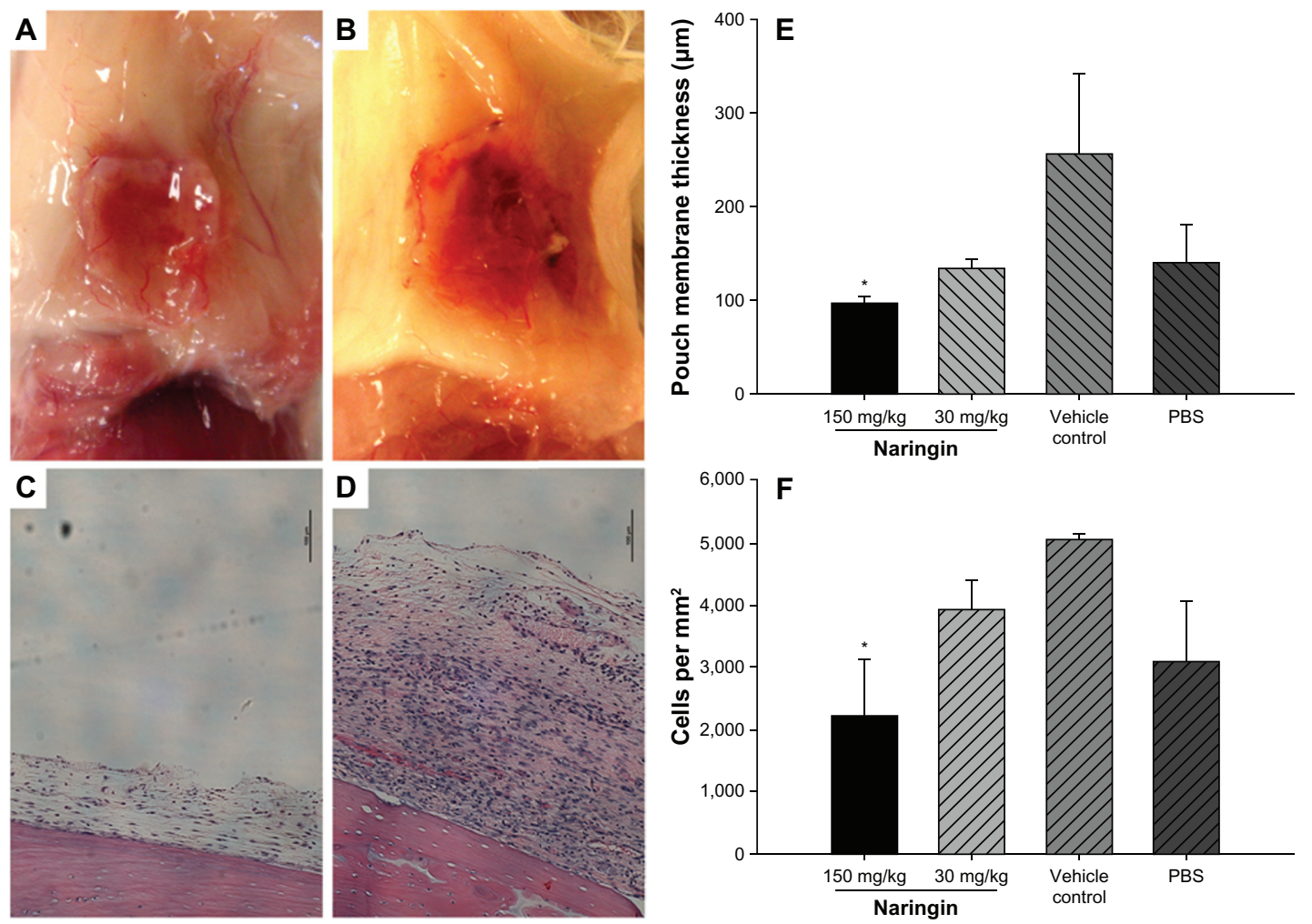

Figure 4 Macroscopic appearance of air pouches dissected from mice 7 days after bone implantation.

Notes: (A) Naringin (I50 mg/kg) treated polymethylmethacrylate (PMMA) challenged air pouch with a piece of calvarium implant. (B) Calvarium implanted air pouch with PMMA stimulation and dimethyl sulfoxide vehicle control. (C) Representative hematoxylin and eosin stained sections of bone implanted pouches in naringin treated $(150 \mathrm{mg} / \mathrm{kg})$ pouch showing ameliorated air pouch membrane (I00X). (D) Representative hematoxylin and eosin stained sections of bone implanted pouches in a pouch containing PMMA debris showing proliferated pouch membrane and extensive inflammatory cell infiltration (I00x). (E) Pouch membrane thickness and (F) total cell counts among groups were summarized by a computerized image analysis system. *Denotes $P<0.05$.

Abbreviation: PBS, phosphate-buffered saline. 

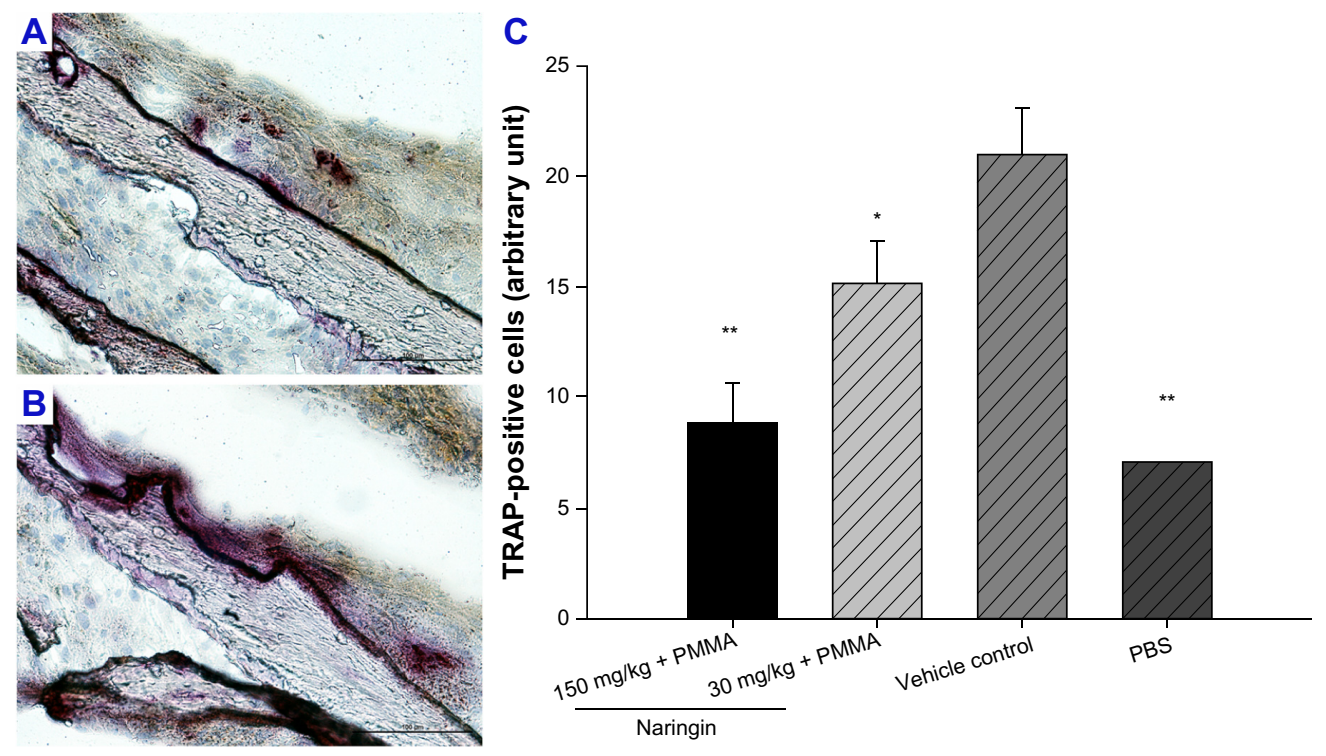

Figure 5 Tartrate resistant acid phosphatase staining on murine pouch with calvaria implantation at 7 days $(200 \times)$.

Notes: (A) Calvarium containing pouch with naringin treatment ( $150 \mathrm{mg} / \mathrm{kg}$ ). (B) Calvarium pouch with polymethylmethacrylate stimulation. (C) Quantification of tartrate resistant acid phosphatase positive cells among the groups. *Denotes $P<0.05$; **denotes $P<0.0$ I.

Abbreviations: PMMA, polymethylmethacrylate; TRAP, tartrate resistant acid phosphatase; PBS, phosphate-buffered saline.

nificantly reduced this inflammatory response (Figure 4C). Pouch membranes with naringin treatment $(150 \mathrm{mg} / \mathrm{kg})$ exhibited mean thickness of $96.18 \pm 7.67 \mu \mathrm{m}$, which was significantly less than those pouches retrieved from untreated animals $(256.69 \pm 84.82 \mu \mathrm{m}, P<0.01)$ (Figure 4E). Naringin treatment also diminished the cell density in the pouch membranes when compared to untreated PMMA particle controls (2219.42 \pm 914.13 per $\mathrm{mm}^{2}$ and 5067.13 \pm 85.60 per $\mathrm{mm}^{2}$, respectively) (Figure 4F).

Histochemical TRAP staining on the calvaria pouch model revealed ubiquitous dark purple stained osteoclast cells accumulated along the bone-membrane interface. While Figure 5B illustrates the $\mathrm{TRAP}^{+}$cell aggregation and erosion pits in PMMA stimulated bone pouch, there were only small numbers of $\mathrm{TRAP}^{+}$cells on naringin $(150 \mathrm{mg} / \mathrm{kg})$ treated specimens (Figure 5A). Figure 5C summarizes the naringin effects on $\mathrm{TRAP}^{+}$cell accumulation assessed by computerized image analysis. Naringin significantly decreased the amount of $\mathrm{TRAP}^{+}$cellular infiltration in comparison with the PMMA control group.

\section{Naringin protected against long-term bone resorption induced by PMMA particles in the mouse proximal tibia pin-implantation model}

The mice tolerated the proximal tibia Ti-pin implantation surgery well and ambulated with the implanted limbs after surgery. Daily intragastric gavage and the injection of PMMA particles into the prosthetic knee appeared to exert no influence on their daily activity.

MicroCT analysis suggested that oral administration (gavages) of naringin protected against the particleinduced periprosthetic bone loss. At 7 weeks after surgery, microCT assessment indicated that the average BV/TV value at the peri-implant region was $43.82 \pm 18.28$ in the $300 \mathrm{mg} / \mathrm{kg}$ naringin treatment group, which was significantly higher than the mean value of $14.10 \pm 0.48$ in the PBS control group $(P<0.05)$. Although there was a therapeutic trend for the $1500 \mathrm{mg} / \mathrm{kg}$ naringin dosage group $(28.30 \pm 10.14)$, no statistical significance was reached in the current study.

The implanted pin pullout test was performed to examine the mechanical stability of the titanium implant post-PMMA particle challenge and naringin treatment. The average peak interfacial shear strength against pulling was $10.92 \pm 1.16,15.21 \pm 3.46$, and $6.71 \pm 2.90$ Newton in the $1500 \mathrm{mg} / \mathrm{kg}$ naringin, $300 \mathrm{mg} / \mathrm{kg}$ naringin, and PBS control group, respectively. Naringin treatment with $300 \mathrm{mg} / \mathrm{kg}$ gavage dosage significantly increased the implant stability (Figure 6D).

Histological evaluation of sections of prosthetic tibiae with titanium particle challenges revealed ubiquitous presence of inflammatory fibrous membranes at the bone-implant interface. However, the interface membranes in sections from naringin treatment groups were markedly thinner or even absent in some samples. Panels A to $\mathrm{C}$ of Figure 7 illustrate the typical cross-sectioned histological appearance 

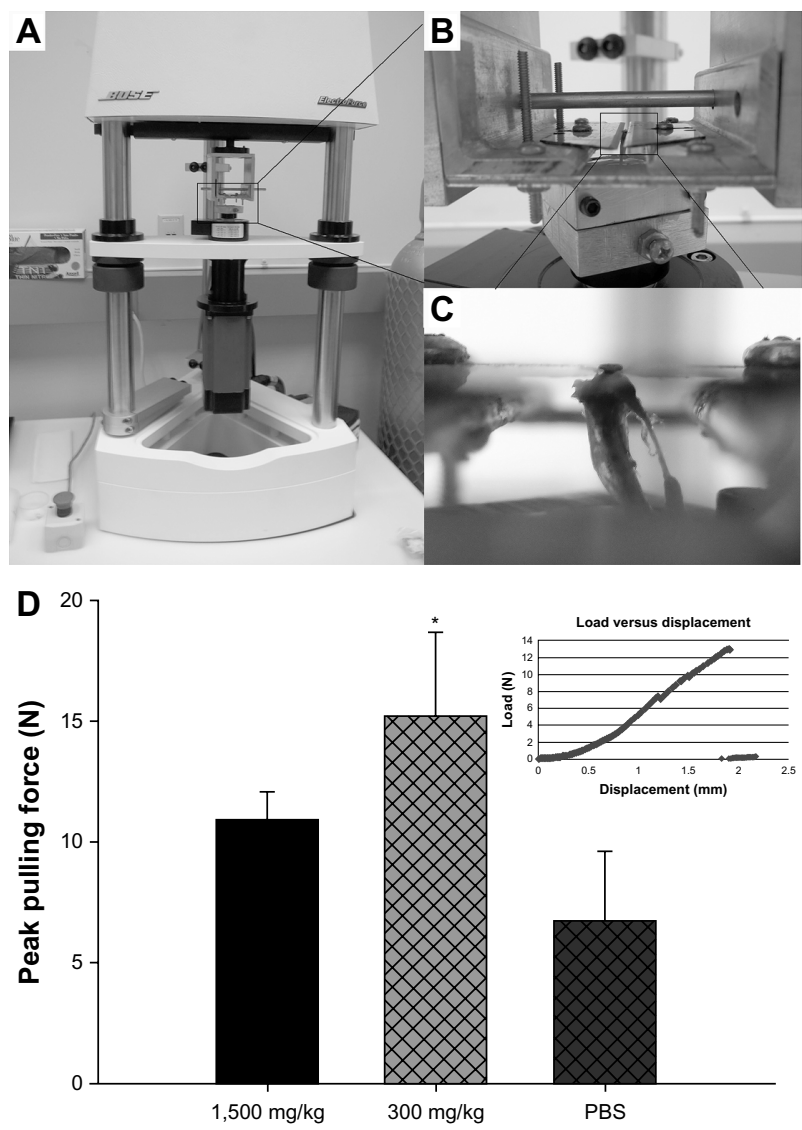

Figure 6 Implanted pin pullout testing.

Notes: (A) The Bose system and assembly used for pin pullout test. (B) The custom designed aluminum fixture to hold and pull the pin out of proximal tibiae. (C) A magnified image of the pin being held with two thin blades. (D) The average pulling force required to pull out the implants among treatment groups (*denotes $P<0.05$ ). Panel (D) illustrates the examples of actual traces of the pulling forces applied to dissociate the titanium pin from the surrounding bone.

of the proximal tibiae with peri-implant pseudomembrane formation. Figure 7D summarizes the measurements of the membrane thickness. The data clearly indicated that both daily lavage dosages of naringin significantly protected against the formation of the inflammatory periprosthetic membrane.

\section{Discussion}

Osteoclast differentiation, or osteoclastogenesis, is a multistep event, which includes the proliferation of osteoclast precursor cells, commitment of cells to the osteoclast lineage, expression of osteoclast-specific genes such as TRAP, and fusion into multinucleated cells. Many studies have indicated that inflammatory osteoclastogenesis is an important step in the development of aseptic loosening. ${ }^{21,22}$ Wear debris, including PMMA, ${ }^{23}$ ultra high molecular weight polyethylene, ${ }^{24}$ and cobalt-chromium and titanium alloys ${ }^{25}$ have been shown to exert a dramatic influence in promoting osteoclastogenesis and/or increasing the numbers of osteoclasts.
PMMA particles alone may be responsible for triggering focal bone resorption. ${ }^{26}$ Studies have shown that PMMA particles were more potent in causing bone resorption than high density polyethylene particles, at least in vitro. ${ }^{27}$ However, Skoglund and Aspenberg ${ }^{28}$ reported bone formation after applying PMMA particles onto a rat bone surface. They hypothesized that the particles may have been inactivated by opsonization. Another explanation may be that the particle size in that study ranged between 5 and $10 \mu \mathrm{m}$, which is at the upper limit for macrophage phagocytosis, ${ }^{29}$ whereas the particles we used were much smaller (mean size of $0.3 \mu \mathrm{m}$ ), causing a stronger cellular response. ${ }^{30}$

Naringin, an active ingredient identified in citrus fruits, has recently been demonstrated to have pharmacological effects in bone protection. Research using an ovariectomized (OVX) mouse model reported that naringin exerted estrogen-like activities to improve general bone properties. ${ }^{31}$ It has also been postulated that naringin may reduce bone resorption by abrogation of osteoclastogenesis via the inhibition of RANKL-induced NF- $\kappa \mathrm{B}$ and extracellular signal-regulated kinase activation. ${ }^{11}$ Naringin is also reported to promote bone formation in a titanium particleinduced diabetic murine calvarial osteolysis model. ${ }^{32} \mathrm{We}$ have recently reported that naringin effectively promoted osteoblast formation and reversed OVX-induced osteoporosis. ${ }^{33}$ In this study we intended to evaluate the potential therapeutic influence of naringin on wear debris associated osteolysis. The data clearly demonstrated that naringin profoundly diminished calcium release from calvaria in the PMMA particle activated mouse RAW 264.7 cell cultures (in vitro) and in the inflammatory environment of the murine air pouch. The inhibitory effect of naringin on osteoclastic bone resorption was confirmed by TRAP staining of implanted calvaria bones. Further, we have shown that the addition of PMMA particles to cultured RAW 264.7 cells can stimulate osteoclastogenesis, while naringin treatment significantly abrogated that activity. It appears that the effects of naringin may be associated with the down-regulation of RANK, TRAP, and CPK gene transcription. Pretreatment of RAW 264.7 cells with naringin at $10 \mu \mathrm{g} / \mathrm{mL}$ significantly reduced the mRNA copy numbers when compared to the controls. While RANK is a receptor essential to control osteoclastogenesis, ${ }^{5} \mathrm{CPK}$ is a cysteine protease that plays a critical role in osteoclastic bone resorption and bone collagen degradation. CPK deficient mice have a mild osteopetrosis and a high number of osteoclasts with impaired function. ${ }^{34}$ These findings suggest that the action of naringin in preventing osteoclastic bone 

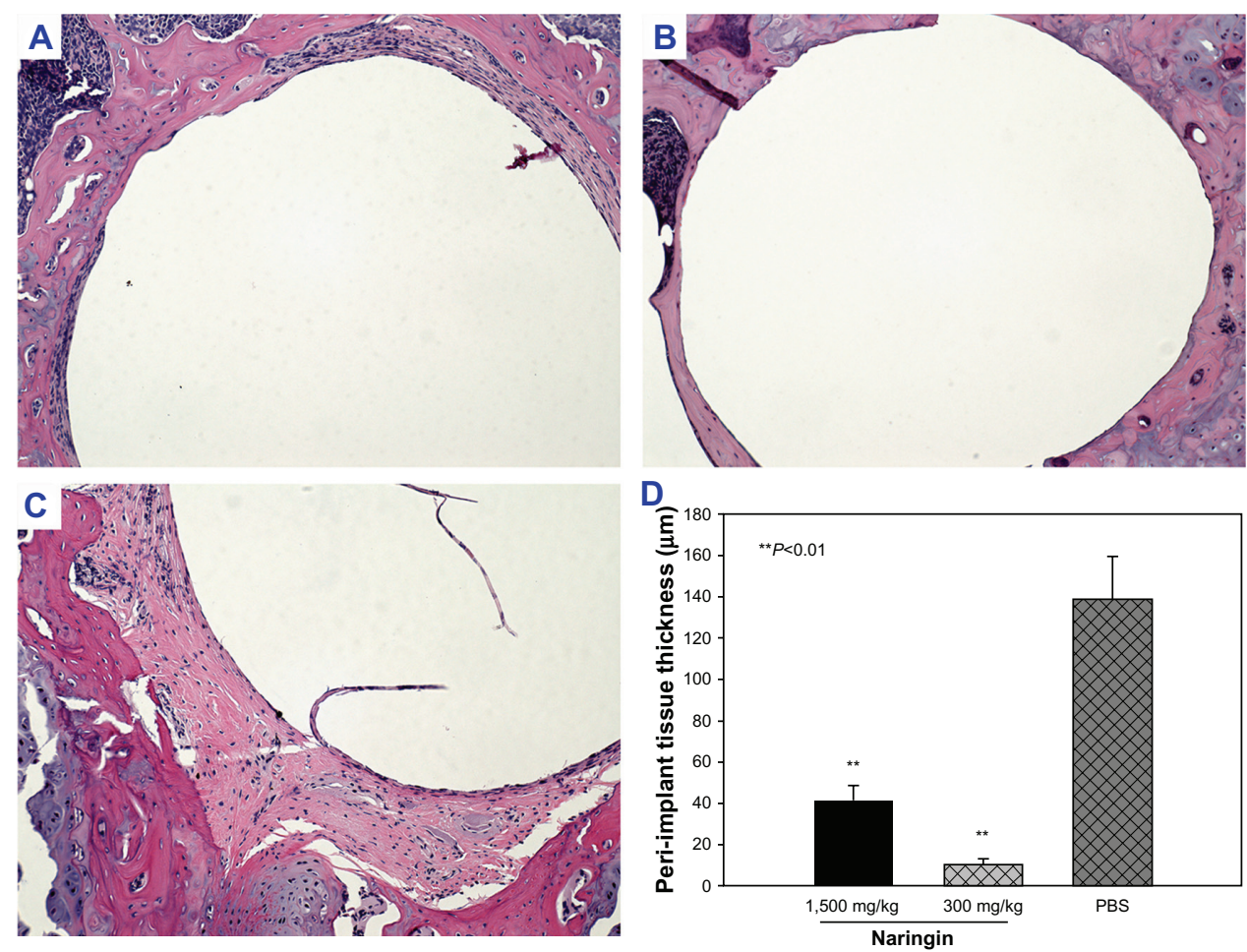

Figure 7 Histological appearance of the pin implanted tibiae at 4 weeks after naringin or phosphate-buffered saline interventions.

Notes: (A) Naringin at I,500 mg/kg; (B) naringin at $300 \mathrm{mg} / \mathrm{kg}$; (C) vehicle control; and (D) quantification summary of the periprosthetic soft tissue thickness among groups (**denotes $P<0.01$ ).

Abbreviation: PBS, phosphate-buffered saline.

resorption as demonstrated in this experiment is mediated through the RANKL/osteoprotegerin pathway.

The air pouch model with bone implantation and particle stimulation developed in our laboratory provides a particle provoked synovial-like membrane that closely surrounds and interacts with implanted bone. ${ }^{15}$ Although very intriguing data have been obtained using this model on the therapeutic effects of naringin including inhibition of PMMA induced local inflammation and protection against bone calcium release, the major limitation of this model is the lack of blood supply to implanted bone which limits the model for a short-term study. We thus further examined the long-term therapeutic influence of naringin using the mouse model of knee pin-implantation failure. After daily oral administration for 30 days, naringin treatment effectively protected against PMMA particle induced periprosthetic soft tissue formation (Figure 7) and surrounding osteolysis (evidenced by microCT bone volume assessment).

Interfacial strength is a major determinant of implant stability and testing the interfacial strength by a biomechanical pullout test represents a useful means to evaluate implant fixation. The interfacial shear load values, peak, and its duration were influenced by bone bonding and provide an assessment of implant stability. ${ }^{35}$ We have successfully developed a pullout test for the mouse pin-implantation model, and previous studies have demonstrated that wear debris particles dramatically decrease the interfacial shear strength and result in the implant loosening. ${ }^{16,36}$ The data from this study suggested that daily oral gavages of naringin appeared beneficial in maintaining the implant stability, although only the lower dose treatment group reached the statistical significance level. Further investigation with increased sample size (animal numbers) is warranted to confirm this data. In addition, although there was no obvious side effects noticed during this 4 week daily treatment period and all animals sustained the gavage and naringin well, long-term safety evaluation of the naringin treatment will be determined.

Many studies have focused on the treatment dosages of naringin administered in vitro and in vivo. A concentration series of naringin was examined in vitro to assess its therapeutic effects. Based on our previous investigation on naringin, ${ }^{33}$ $30 \mathrm{mg} / \mathrm{kg}$ and $150 \mathrm{mg} / \mathrm{kg}$ concentrations were used on air pouch injection and DMSO was used as vehicle to increase its solubility. To evaluate the long-term therapeutic influence of the compound for PMMA induced prosthetic implant loosening, daily oral gavage was given to administer naringin at $1500 \mathrm{mg} / \mathrm{kg}$ and $300 \mathrm{mg} / \mathrm{kg}$, respectively. It is arguable to use 
oral administration of naringin because naringin undergoes some metabolic process after oral administration. According to one study, ${ }^{37}$ about $21 \%$ of administered naringin was recovered in rat excreta in the form of naringin, total naringenin, and 4-hydroxyphenylpropionic acid, and about 60\% was recovered in dog excreta. So naringin metabolites such as naringenin and naringenin glucuronide could be a possible authentic cause of the pharmaceutical effects. However, the current study suggested that daily gavages of naringin effectively protected against PMMA induced osteolysis and the prosthetic pin loosening.

In conclusion, this is the first report to our knowledge that demonstrates that naringin can effectively protect against particle induced osteoclastogenesis and bone degradation in both short- and long-term animal models of osteolysis. The data suggested that the influence of naringin may act through the down-regulation of RANK, TRAP, and CPK pathways. Our findings suggest that naringin may represent a potential agent for the prevention and treatment of aseptic loosening. Further studies are indeed warranted to evaluate its pharmacokinetics, toxicology, and the drug delivery strategies.

\section{Acknowledgments}

This work was supported in part by a research grant from Wichita Medical Research and Education Foundation (WMREF), a research grant from Shandong Provincial Natural Science Foundation, People's Republic of China (No Y2008C147), and research funds from Orthopaedic Research Institute, Via Christi Wichita Hospitals Inc. The authors wish to thank Ms Zheng Song for her technical assistance.

\section{Disclosure}

The authors declare no conflict of interest in this work.

\section{References}

1. Holt G, Murnaghan C, Reilly J, Meek RM. The biology of aseptic osteolysis. Clin Orthop Relat Res. 2007;460:240-252.

2. Purdue PE, Koulouvaris P, Potter HG, Nestor BJ, Sculco TP. The cellular and molecular biology of periprosthetic osteolysis. Clin Orthop Relat Res. 2007;454:251-261.

3. Morrissey C, Kostenuik PL, Brown LG, Vessella RL, Corey E. Hostderived RANKL is responsible for osteolysis in a C4-2 human prostate cancer xenograft model of experimental bone metastases. BMC Cancer. 2007;7:148.

4. Bell NH. RANK ligand and the regulation of skeletal remodeling. J Clin Invest. 2003;111(8):1120-1122.

5. Li J, Sarosi I, Yan XQ, et al. RANK is the intrinsic hematopoietic cell surface receptor that controls osteoclastogenesis and regulation of bone mass and calcium metabolism. Proc Natl Acad Sci U S A. 2000;97(4): $1566-1571$

6. Troen BR. The role of cathepsin K in normal bone resorption. Drug News Perspect. 2004;17(1):19-28.
7. Konttinen YT, Takagi M, Mandelin J, et al. Acid attack and cathepsin $\mathrm{K}$ in bone resorption around total hip replacement prosthesis. J Bone Miner Res. 2001;16(10):1780-1786.

8. Wei M, Yang Z, Li P, Zhang Y, Sse WC. Anti-osteoporosis activity of naringin in the retinoic acid-induced osteoporosis model. Am J Chin Med. 2007;35(4):663-667.

9. Zhang P, Dai KR, Yan SG, et al. Effects of naringin on the proliferation and osteogenic differentiation of human bone mesenchymal stem cell. Eur J Pharmacol. 2009;607(1-3):1-5.

10. Hirata M, Matsumoto C, Takita M, Miyaura C, Inada M. Naringin suppresses osteoclast formation and enhances bone mass in mice. Journal of Health Science. 2009;55(3):463-467.

11. Ang ES, Yang X, Chen H, Liu Q, Zheng MH, Xu J. Naringin abrogates osteoclastogenesis and bone resorption via the inhibition of RANKLinduced NF-kappaB and ERK activation. FEBS Lett. 2011;585(17): 2755-2762.

12. Wooley PH, Morren R, Andary J, et al. Inflammatory responses to orthopaedic biomaterials in the murine air pouch. Biomaterials. 2002;23(2):517-526.

13. Karsdal MA, Henriksen K, Sorensen MG, et al. Acidification of the osteoclastic resorption compartment provides insight into the coupling of bone formation to bone resorption. Am J Pathol. 2005;166(2): 467-476.

14. Ren W, Wu B, Mayton L, Wooley PH. Polyethylene and methyl methacrylate particle-stimulated inflammatory tissue and macrophages up-regulate bone resorption in a murine neonatal calvaria in vitro organ system. J Orthop Res. 2002;20(5):1031-1037.

15. Ren W, Yang SY, Wooley PH. A novel murine model of orthopaedic wear-debris associated osteolysis. Scand J Rheumatol. 2004;33(5): 349-357.

16. Yang SY, Yu H, Gong W, et al. Murine model of prosthesis failure for the long-term study of aseptic loosening. J Orthop Res. 2007;25(5): 603-611.

17. Croke M, Ross FP, Korhonen M, Williams DA, Zou W, Teitelbaum SL. Rac deletion in osteoclasts causes severe osteopetrosis. J Cell Sci. 2011;124(Pt 22):3811-3821.

18. Tsuchiya S, Simmer JP, Hu JC, Richardson AS, Yamakoshi F, Yamakoshi Y. Astacin proteases cleave dentin sialophosphoprotein (Dspp) to generate dentin phosphoprotein (Dpp). J Bone Miner Res. 2011;26(1):220-228.

19. Yang SY, Wu B, Mayton L, et al. Protective effects of IL-1Ra or vIL-10 gene transfer on a murine model of wear debris-induced osteolysis. Gene Ther. 2004;11(5):483-491.

20. Yang SY, Mayton L, Wu B, Goater JJ, Schwarz EM, Wooley PH. Adeno-associated virus-mediated osteoprotegerin gene transfer protects against particulate polyethylene-induced osteolysis in a murine model. Arthritis Rheum. 2002;46(9):2514-2523.

21. Childs LM, Paschalis EP, Xing L, et al. In vivo RANK signaling blockade using the receptor activator of NF-kappaB:Fc effectively prevents and ameliorates wear debris-induced osteolysis via osteoclast depletion without inhibiting osteogenesis. J Bone Miner Res. 2002;17(2): 192-199.

22. Greenfield EM, Bi Y, Ragab AA, Goldberg VM, Van De Motter RR. The role of osteoclast differentiation in aseptic loosening. J Orthop Res. 2002;20(1):1-8.

23. Zhang H, Ricciardi BF, Yang X, Shi Y, Camacho NP, Bostrom MG. Polymethylmethacrylate particles stimulate bone resorption of mature osteoclasts in vitro. Acta Orthop. 2008;79(2):281-288.

24. De Man FH, Tigchelaar W, Marti RK, Van Noorden CJ, Van der Vis HM. Effects of mechanical compression of a fibrous tissue interface on bone with or without high-density polyethylene particles in a rabbit model of prosthetic loosening. J Bone Joint Surg Am. 2005;87(7):1522-1533.

25. MacQuarrie RA, Fang Chen Y, Coles C, Anderson GI. Wearparticle-induced osteoclast osteolysis: the role of particulates and mechanical strain. J Biomed Mater Res B Appl Biomater. 2004;69(1): 104-112. 
26. Willert HG, Bertram H, Buchhorn GH. Osteolysis in alloarthroplasty of the hip. The role of bone cement fragmentation. Clin Orthop Relat Res. 1990(258):108-121.

27. Pandey R, Quinn J, Joyner C, Murray DW, Triffitt JT, Athanasou NA. Arthroplasty implant biomaterial particle associated macrophages differentiate into lacunar bone resorbing cells. Ann Rheum Dis. 1996;55(6):388-395.

28. Skoglund B, Aspenberg P. PMMA particles and pressure - a study of the osteolytic properties of two agents proposed to cause prosthetic loosening. J Orthop Res. 2003;21(2):196-201.

29. Horowitz SM, Doty SB, Lane JM, Burstein AH. Studies of the mechanism by which the mechanical failure of polymethylmethacrylate leads to bone resorption. J Bone Joint Surg Am. 1993;75(6):802-813.

30. Green TR, Fisher J, Stone M, Wroblewski BM, Ingham E. Polyethylene particles of a 'critical size' are necessary for the induction of cytokines by macrophages in vitro. Biomaterials. 1998;19(24):2297-2302.

31. Pang WY, Wang XL, Mok SK, et al. Naringin improves bone properties in ovariectomized mice and exerts oestrogen-like activities in rat osteoblastlike (UMR-106) cells. Br J Pharmacol. 2010;159(8):1693-1703.
32. Zhou $\mathrm{X}$, Zhang $\mathrm{P}$, Zhang $\mathrm{C}$, Zhu Z. Promotion of bone formation by naringin in a titanium particle-induced diabetic murine calvarial osteolysis model. J Orthop Res. 2010;28(4):451-456.

33. Li N, Jiang Y, Wooley PH, Xu Z, Yang SY. Naringin promotes osteoblast differentiation and effectively reverses ovariectomy-associated osteoporosis. J Orthop Sci. 2013;18(3):478-485.

34. Pennypacker B, Shea M, Liu Q, et al. Bone density, strength, and formation in adult cathepsin K (-/-) mice. Bone. 2009;44(2): 199-207.

35. Chang YS, Kobayashi M, Li ZL, Oka M, Nakamura T. Significance of peak value and duration of the interfacial shear load in evaluation of the bone-implant interface. Clin Biomech (Bristol, Avon). 2003;18(8): 773-779.

36. Zhang T, Yu H, Gong W, et al. The effect of osteoprotegerin gene modification on wear debris-induced osteolysis in a murine model of knee prosthesis failure. Biomaterials. 2009;30(30):6102-6108.

37. Liu M, Zou W, Yang C, Peng W, Su W. Metabolism and excretion studies of oral administered naringin, a putative antitussive, in rats and dogs. Biopharm Drug Dispos. 2012;33(3):123-134.

\section{Publish your work in this journal}

Drug Design, Development and Therapy is an international, peerreviewed open-access journal that spans the spectrum of drug design and development through to clinical applications. Clinical outcomes, patient safety, and programs for the development and effective, safe, and sustained use of medicines are a feature of the journal, which has also been accepted for indexing on PubMed Central. The manuscript management system is completely online and includes a very quick and fair peer-review system, which is all easy to use. Visit http://www.dovepress.com/testimonials.php to read real quotes from published authors

Submit your manuscript here: http://www.dovepress.com/drug-design-development-and-therapy-journal 